\title{
Curettage after uterine artery embolization combined with methotrexate treatment for caesarean scar pregnancy
}

\author{
YING FENG $^{1}$, SUWEN CHEN $^{1}$, CHANGDONG LI $^{1}$, XIAOFENG ZHANG ${ }^{1}$, \\ HUA DUAN $^{1}$, SUREN SOORANNA ${ }^{2}$, MARK R. JOHNSON ${ }^{2}$ and JIAN LI ${ }^{1}$ \\ ${ }^{1}$ Department of Gynaecology, Beijing Obstetrics and Gynaecology Hospital, Capital Medical University, \\ Beijing 100026, P.R. China; ${ }^{2}$ Department of Gynaecology, Imperial College London, \\ Chelsea and Westminster Hospital, London SW10 9NH, UK
}

Received March 9, 2016; Accepted June 29, 2016

DOI: $10.3892 /$ etm.2016.3489

\begin{abstract}
In the present study, we evaluated the diagnosis and management modalities of caesarean scar pregnancy (CSP). Thirty patients diagnosed with CSP were retrospectively studied between February, 2010 and February, 2012. Twenty-five patients were offered prophylactic uterine artery embolization (UAE) and methotrexate (MTX) prior to uterine suction curettage. Five cases were referred from other hospitals where the initial management with uterine suction curettage had resulted in uncontrollable massive haemorrhage, 4 of the cases had UAE and one proceeded immediately to hysterectomy. In the 25 patients treated with prophylactic UAE and MTX, 12 had laparoscopy-guided curettage and 13 had ultrasound-guided curettage without complication. The results showed that the 25 patients with CSP, who received prophylactic UAE and MTX prior to uterine curettage, recovered without complications. Five patients referred from other hospitals, where uterine curettage was the primary procedure, had severe complications including uncontrolled vaginal bleeding and uterine rupture. Four of the five patients were treated successfully with emergency UAE and the remaining patient underwent emergency hysterectomy as ultrasound examination detected significant haemorrhage between the uterus and the bladder. Of the 25 patients who received prophylactic UAE combined with MTX, there were no reports of irregular menstruation or serious adverse effects. Notably, the decrease in serum human chorionic gonadotropin (HCG) levels 3 days post-surgery was greater with ultrasound-guided curettage $(84.3 \pm 5.5 \%)$ than with laparoscopy-guided curettage $(76.3 \pm 10.2 \%)$. In summary, the data suggested that
\end{abstract}

Correspondence to: Dr Jian Li, Department of Gynaecology, Beijing Obstetrics and Gynaecology Hospital, Capital Medical University, 251 Yao Iiayuan Road, Beijing 100026, P.R. China E-mail: 1ijian02@hotmail.com

Key words: caesarean scar pregnancy, uterine artery embolization, methotrexate, suction curettage, human chorionic gonadotrophin prophylactic UAE with MTX followed by ultrasound-guided curettage is the most effective therapeutic approach in CSP.

\section{Introduction}

Caesarean scar pregnancy (CSP) is an uncommon but serious complication of a previous caesarean. The first case of CSP was reported in the medical literature in 1978 by Larsen and Solomon (1) and the reported incidence of CSP was approximately $0.15 \%$ of ectopic pregnancies (2). Since 2000 , the incidence of CSP has increased significantly to $6.1 \%$ of all ectopic pregnancies in those with previous caesarean sections, which may reflect the increasing number of caesarean sections being performed and the improved accuracy of diagnosis of ectopic pregnancies using colour Doppler transvaginal ultrasonography (3). In addition, the rates of CSP may have increased as a result of the use of assisted reproduction technology (4). Typically the diagnosis was made at between 6 and 9 weeks gestation.

CSP was confirmed by a thorough medical history evaluation, including obstetric, reproductive and surgical history, a physical examination and increased levels of progesterone and total serum human chorionic gonadotropin (HCG) between 6 and 9 weeks of gestation. The diagnosis of CSP was mainly accomplished by combining transvaginal sonography (TVS) with Doppler flow imaging (5).

There is no standard treatment for the management of CSP and only a few cases have been reported in the literature. Although CSP is rare, without a high index of suspicion and early diagnosis, it may result in serious maternal morbidity, including uterine rupture and haemorrhage, or mortality (6). Termination of pregnancy in the first trimester is strongly recommended to prevent life-threatening complications and to maintain the possibility of future pregnancies (7). In the past, the preferred treatment option was to perform a laparotomy with the possible need for hysterectomy to avoid potential lethal haemorrhage (8). The current methods for the management of CSP include systemic chemotherapy with methotrexate (MTX), local injection of embryocidic agents, uterine curettage, hysteroscopic evacuation, laparoscopic management, excision of the involved lower segment of the uterus, uterine artery embolization (UAE) and expectant 
Table I. Characteristics of the clinical cases.

\begin{tabular}{lccccc}
\hline Values & Age (years) & $\begin{array}{c}\text { No. of previous } \\
\text { caesarean sections }\end{array}$ & No. of pregnancies & $\begin{array}{c}\text { Interval time from recent } \\
\text { caesarean section (years) }\end{array}$ & Gestation (weeks) \\
\hline Mean & $32.20 \pm 4.83$ & $1.20 \pm 0.61$ & $3.60 \pm 1.55$ & $4.45 \pm 1.34$ & $8.34 \pm 3.70$ \\
Range (min - max) & $23-43$ & $1-4$ & $2-7$ & 6 months-12 years & $5-12$ \\
\hline
\end{tabular}

management $(9,10)$. In the present study, we reported the outcome of 30 cases of CSP managed by using uterine curettage as a primary approach or combined with prophylactic UAE and MTX prior to uterine curettage. In the 25 cases managed with prophylactic UAE and MTX, we compared CSP removal using ultrasound-guided uterine curettage and laparoscopy-guided curettage.

\section{Patients and methods}

Patients. Thirty patients with CSP were treated with uterine curettage with or without prophylactic UAE and MTX. These cases were identified from the Beijing Obstetrics and Gynaecology Hospital database and were analysed retrospectively. The characteristics of the 30 patients are shown in Table I. Clinical data were analyzed with respect to age, gravidity and parity, history of caesarean delivery, interval from the most recent caesarean delivery to diagnosis, clinical presentation, results of laboratory examination, process of diagnosis and treatment sequence, and outcome, which were collected from the original hospital charts, operation notes and outpatient medical records via telephone questionnaires.

At Beijing Obstetrics and Gynaecology Hospital, transabdominal ultrasound with full bladder was performed initially to assess the pelvis and uterus with careful inspection of the interface between the anterior lower uterine segment and bladder. This was followed by a transvaginal ultrasound to allow for the fine-detail evaluation of the gestational sac in relation to the scar. The diagnosis of CSP in the first trimester was determined based on the following ultrasonographic criteria: i) an empty uterus with a clearly visualized endometrium; ii) an empty cervical canal; iii) an anteriorly located gestational sac with a decreased myometrium layer between the bladder and the sac; and iv) a reduced or absent myometrium between the gestational sac and bladder on a sagittal view of the uterus (this was observed to be $<5 \mathrm{~mm}$ in two-thirds of cases) (11) (Fig. 1).

Written informed consent was obtained prior to UAE from each participant with CSP. Approval for the study was obtained from the ethics committee of the Beijing Obstetrics and Gynaecology Hospital.

Procedure. Twenty-five patients with a clear diagnosis of CSP were offered prophylactic UAE prior to uterine curettage. A right transfemoral approach was used for artery access, and each uterine artery was selectively catheterized with a 4- or 5-F Roberts uterine catheter. Prior to UAE, $50 \mathrm{mg}$ of MTX was dissolved in $20 \mathrm{ml}$ of physiologic saline solution. This dose was separated between the two uterine arteries and infused via the arterial catheter. UAE was performed by an experienced radiologist with the use of gelfoam particles
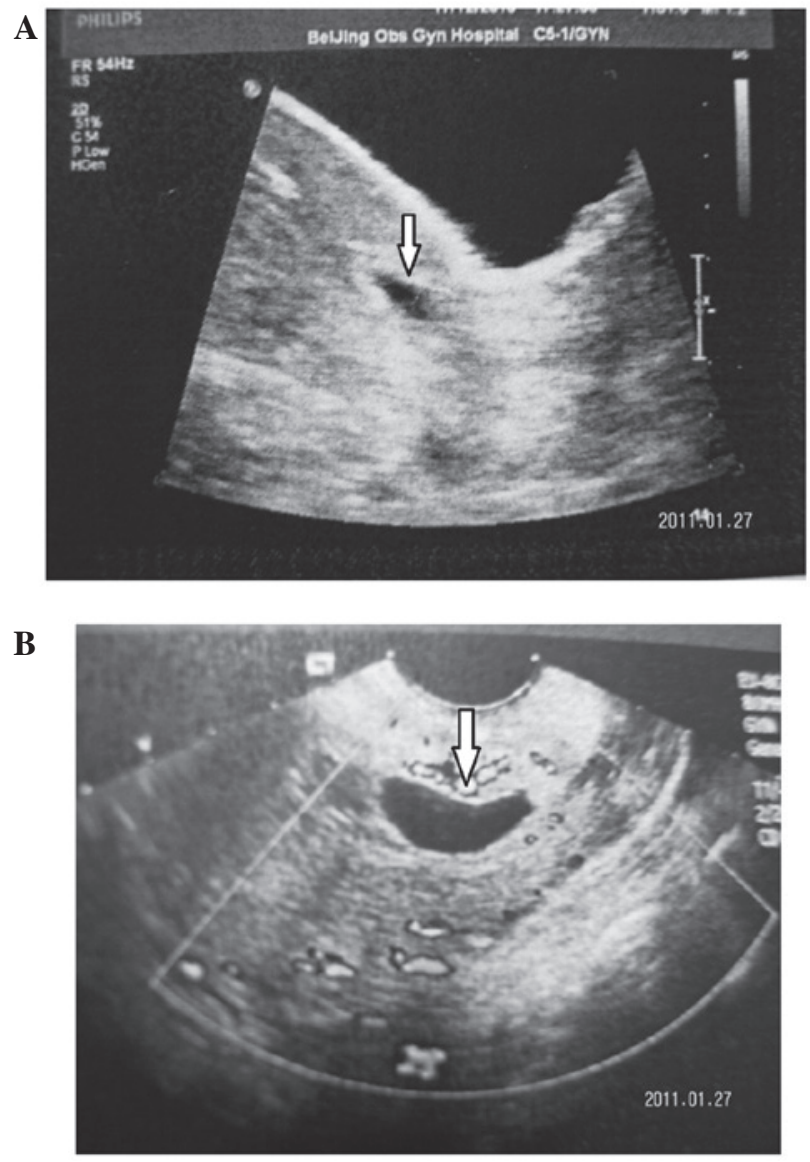

Figure 1. Two examples of echo-images of CSP. Images were captured from a 30-year-old woman at 6 weeks of CSP with a history of one caesarean delivery. (A) TAS showing the midline of the uterus. (B) Transverse TVS showing the midline of the uterus. Arrow shows pregnant scar. CSP, caesarean scar pregnancy; TAS, transabdominal sonography; TVS, transvaginal sonography.

(500-1,000 $\mu \mathrm{m}$ in diameter) mixed with nonionic contrast medium. Angiography was performed after UAE to confirm that the bilateral uterine arteries were occluded (Fig. 2). After 24-48 h, uterine curettage was performed under ultrasoundguided or laparoscopy-guided curettage to confirm the complete removal or destruction of the CSP mass. The other five patients, who were misdiagnosed from other hospitals as having an intrauterine pregnancy were treated with emergency UAE because of uncontrollable massive haemorrhage within $48 \mathrm{~h}$ after uterine curettage. Prophylactic anti-infection drugs were administered to prevent infection. Serum HCG levels were measured every three days.

Follow-up. The patients were recommended to use contraception for the first year postoperatively. They were followed up 
Table II. A comparison of the different types of clinical cases.

\begin{tabular}{lccccc}
\hline $\begin{array}{l}\text { Clinical } \\
\text { presentation }\end{array}$ & $\begin{array}{c}\text { Vaginal } \\
\text { bleeding }\end{array}$ & $\begin{array}{c}\text { Abdominal } \\
\text { pain }\end{array}$ & $\begin{array}{c}\text { Vaginal bleeding } \\
\text { abdominal pain }\end{array}$ & $\begin{array}{c}\text { Asymptomatic } \\
\text { Massive bleeding } \\
\text { after curettage }\end{array}$ & $\begin{array}{c}5 \\
16.67 \%\end{array}$ \\
\hline Case & 11 & 1 & 5 & $26.67 \%$ & $16.67 \%$ \\
\hline
\end{tabular}

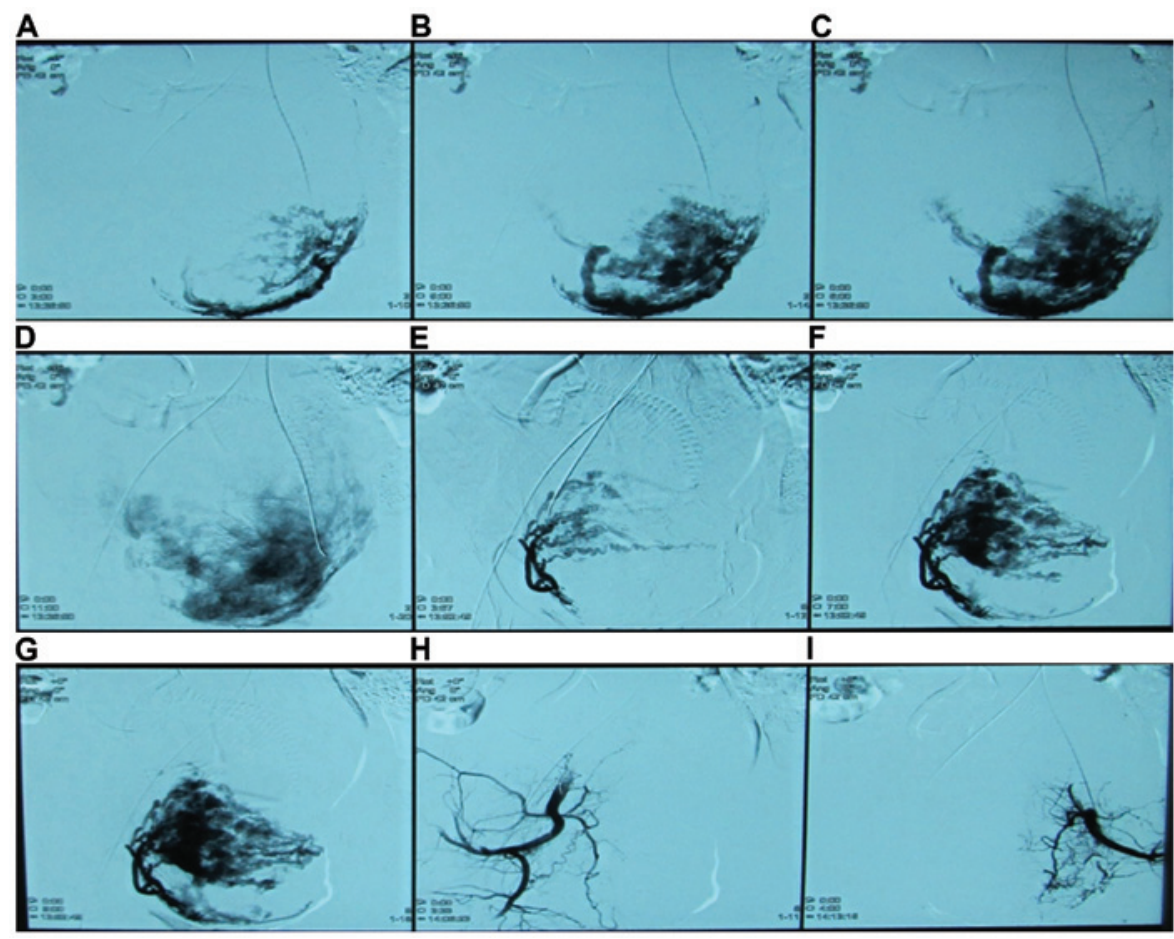

Figure 2. Highly selective uterine artery angiograms. A 30-year-old woman with a history of one caesarean delivery having CSP at 6 weeks underwent preventive UAE. (A-G) Before UAE, selective uterine artery angiograms demonstrated enlarged and tortuous uterine arteries, with a hypervascular region overlying the lower uterine segment corresponding to the CSP region. (H and I) CSP image disappeared after an arterial occlusion was successfully achieved. CSP, caesarean scar pregnancy; UAE, uterine artery embolization.

by telephone interview between three months and one year depending on serum HCG results, ultrasonographic findings and menstrual cycle recovery.

Statistical analysis. Statistical analysis was undertaken using SPSS version 13.0 software (Chicago, IL, USA). Measurement data were presented as the means and standard deviations. The significance of between-group differences was tested using analysis of variance or Chi-square tests. $\mathrm{P}<0.05$ was considered statistically significant. The data were normally distributed $(\mathrm{P}>0.05)$.

\section{Results}

Patients. A total of 30 women were included in the current study. Clinical presentation was described as follows: mild vaginal bleeding (11 patients); mild abdominal pain (1 patient); both of the above present ( 5 patients) and asymptomatic (8 patients; Table II). Twenty-five patients diagnosed for CSP by abdominal and TVS were approached to participate in the study. The patients received prophylactic UAE combined with MTX followed by uterine curettage and all 25 patients recovered without complications. The remaining five patients, who were misdiagnosed as having an intrauterine pregnancy in another hospital, were treated with suction curettage initially complicated by uncontrolled vaginal bleeding. Four of the five patients were treated successfully with UAE in our hospital. Five days after UAE, the patients underwent ultrasound-guided repeat uterine curettage because of a slow decrease in serum $\beta$-HCG or continued vaginal bleeding. The last patient underwent laparotomy and hysterectomy after an expanding haematoma was demonstrated between the uterus and bladder by transabdominal ultrasound (Fig. 3).

Of the 25 patients who received preventive UAE combined with MTX, there were no reports of irregular menstrual or serious adverse effects. Two patients developed postoperative fever but responded to anti-infective treatment administered at the hospital. Of the 25 patients, 12 had laparoscopy-guided curettage and 13 had ultrasound-guided curettage. Of the patients who received laparoscopy-guided curettage, three patients chose the surgical option due to a desire for tube ligation. During follow up, no serious adverse effects were observed in these patients. However, serum $\beta$-HCG levels decreased more rapidly with ultrasound-guided curettage than with laparoscopy-guided 
$\mathbf{A}$

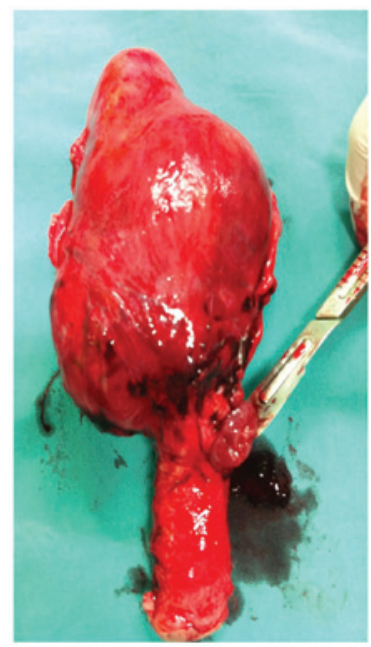

C

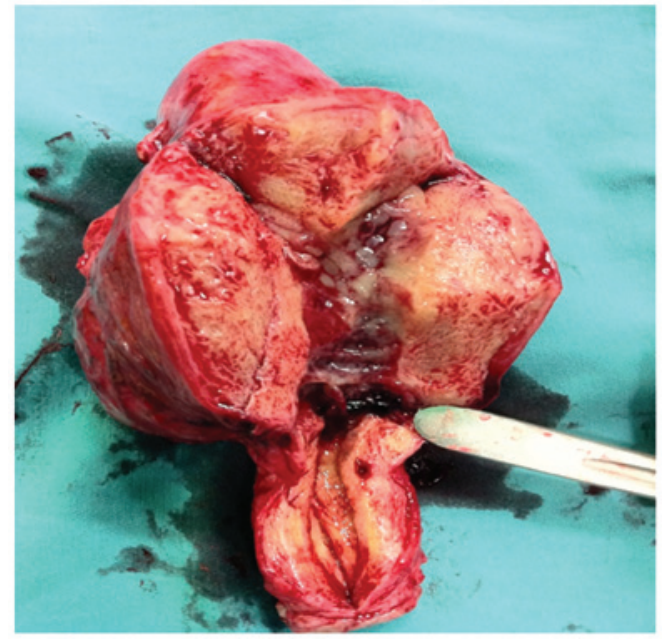

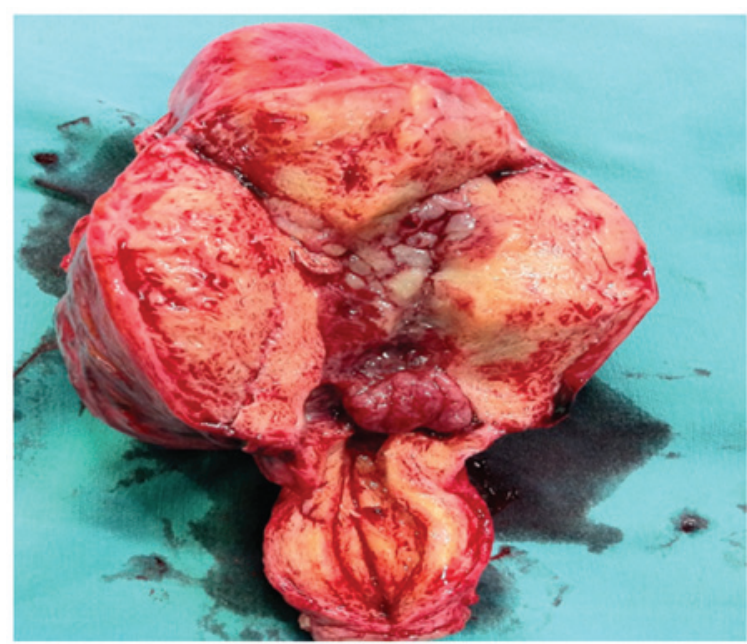

D

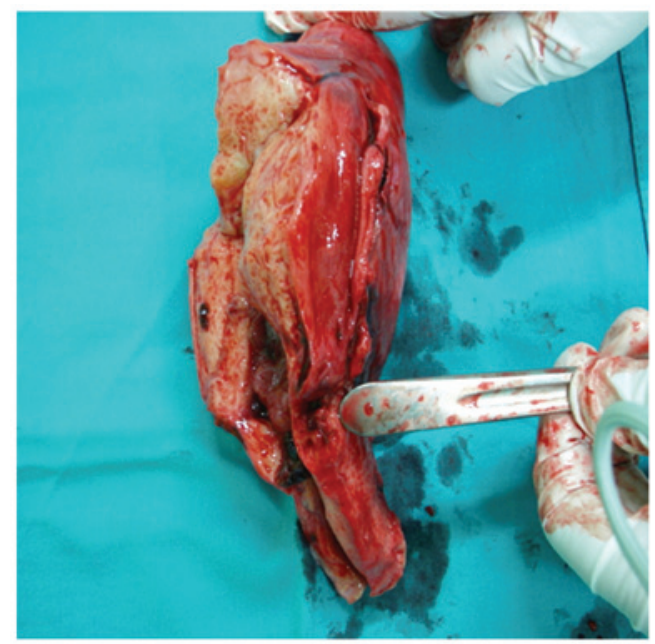

Figure 3. Dissection of uterus with CSP after hysterectomy. A 42-year-old woman with a history of one caesarean delivery had CSP for 8 weeks. She had uncontrolled haemorrhage due to CSP uterus rupture. (A) Whole uterus, (B) the gestational sac implanted into the caesarean scar myometrium, (C) chorionic villi from pregnancy at the caesarean scar, and (D) the sagittal angle of the cavity with respect to the scar tissue. CSP, caesarean scar pregnancy.

curettage. The decreased rate was $84.3 \pm 10.5$ and $76.3 \pm 15.23 \%$, respectively (Table III). In the patients undergoing laparoscopy and ultrasound-guided curettage, the serum $\beta$-HCG levels returned to normal after 25 and 34 days. The mean curettage time was $15 \pm 5.3 \mathrm{~min}$ and mean blood loss was $16 \pm 3.8 \mathrm{ml}$. Ultrasound examination was performed prior to discharge from the hospital to ensure that the caesarean scar had disappeared, and that no abnormal ultrasound findings were present. Follow-up interviews indicated that a normal menstrual cycle had returned $36.9 \pm 2.9$ days postoperatively, with no reports of abnormal vaginal bleeding. None of the patients had normal intrauterine pregnancies during the follow-up period.

\section{Discussion}

CSP is a rare form of ectopic pregnancy. The etiology of CSP is unclear, but it is generally thought that CSP occurs when a blastocyst is implanted on fibrous scar tissue within a wedge-shaped myometrial defect in the anterior lower uterine segment at the site of a prior caesarean scar $(12,13)$. The pathological examination of excised CSP alone and in hysterectomy specimens demonstrated clusters of trophoblast cells as well as scattered syncytiotrophoblast cells invading the myometrium through a microscopic dehiscent tract created by a previous caesarean section procedure or other uterine surgery $(12,13)$.

CSP can present at any time from implantation to term but has been reported to present more commonly in the first trimester. The common presenting symptoms are vaginal bleeding and abdominal pain, but at least one-third of patients are asymptomatic $(6,14)$. The results of the present study indicate one-fourth of patients. In the present study, five patients were misdiagnosed for having an intrauterine pregnancy and had uncontrolled massive hemorrhage after uterine curettage. Therefore, true diagnosis was crucial as a large number of complications caused by misdiagnosis may lead to inappropriate interventions. TVS was the reference standard for the diagnosis of CSP in the first trimester with a reported $86.4 \%$ sensitivity $(12,14)$. Maymon et al (15) recommended using combined TVS and transabdominal sonography (TAS) with a full bladder. Thus a 'panoramic view' of the uterus is provided with accurate measurement of the distance between the gestational sac and bladder (15). In the present study, all 30 patients were diagnosed via a combination of TVS and TAS.

Two different types of CSP have been proposed (16). The first type is an implantation on the prior scar with progression towards the cervico-isthmic space or the uterine cavity. 
Table III. A comparison of operative outcomes between laparoscopy-guided curettage and ultrasound-guided curettage.

\begin{tabular}{|c|c|c|c|c|c|c|}
\hline & $\begin{array}{l}\text { No. of previous } \\
\text { caesarean sections }\end{array}$ & $\begin{array}{l}\text { No. of } \\
\text { previous } \\
\text { abortion }\end{array}$ & $\begin{array}{c}\text { Apart time of } \\
\text { previous caesarean } \\
\text { section (years) }\end{array}$ & $\begin{array}{c}\text { Blood loss } \\
\text { volume (ml) }\end{array}$ & $\begin{array}{l}\text { Menstrual cycle } \\
\text { recovery (days) }\end{array}$ & $\begin{array}{c}\text { Rate of } \\
\text { serum } \beta-\mathrm{HCG} \\
\text { reduction }(\%)^{\mathrm{a}}\end{array}$ \\
\hline $\begin{array}{l}\text { Ultrasound-guided } \\
\text { curettage }\end{array}$ & $1.14 \pm 0.3$ & $1.86 \pm 1.03$ & $3.71 \pm 3.36$ & $17.50 \pm 4.16$ & $37.14 \pm 3.79$ & $88.43 \pm 10.50$ \\
\hline $\begin{array}{l}\text { Laparoscopy-guided } \\
\text { curettage }\end{array}$ & $1.09 \pm 0.30$ & $2.82 \pm 1.83$ & $4.01 \pm 2.59$ & $15.91 \pm 3.36$ & $36.64 \pm 1.36$ & $76.31 \pm 15.23$ \\
\hline $\mathrm{P}$-value & 0.70 & 0.14 & 0.80 & 0.83 & 0.43 & 0.04 \\
\hline
\end{tabular}

${ }^{a}$ The reduction rate of serum $\mathrm{HCG}=$ preoperative serum HCG - postoperative serum HCG (three days after operation)/preoperative serum HCG. HCG, human chorionic gonadotropin.

Such a pregnancy may progress to viability with the risk of massive haemorrhage. The second type is a deep implantation into the caesarean scar defect growing towards the bladder and abdominal cavity and more prone to scar rupture $(11,12)$.

Successful births have been described with the appropriate management, but the prognosis for an uneventful term pregnancy is poor. The hysterectomy rate in these cases is $71 \%$ because of the increased risk of placental previa/accreta and massive hemorrhage (12-17). Termination of the diagnosed cases by surgical or medical means may improve the outcomes by allowing preservation of the uterus and future fertility. It is anticipated that delay in treatment of scar pregnancies can lead to uterine rupture, hysterectomy and significant maternal morbidity $(11,18)$. Various medical and surgical treatments have been attempted, however, there is no consensus on the optimal mode of treatment (19). Many medical and surgical approaches have been attempted with the aim of eliminating the gestational sac and preserving fertility, including systemic MTX or local injection into the gestation sac of MTX, potassium chloride, hyperosmolar glucose or prostaglandins. The surgical methods included dilatation and curettage, UAE, hysteroscopic resection, laparoscopic resection or even hysterectomy. Local, systemic and combined treatments with MTX have been described. However, fibrous tissue within the scar around the gestational sac can delay systemic MTX absorption into the sac (20). Local injection of embryocides under sonographic guidance may decrease the need of additional interventions compared to systemic MTX $(11,12,14)$. However, close monitoring of the patient after intervention is required as hemorrhage may still occur. Therefore, bilateral UAE should be considered $(13,15)$.

Surgical management with laparoscopy or laparotomy with excision of the pregnancy may be optima for women who do not respond to conservative medical treatments or are late to present for medical attention (19). However, surgery carries the risk of postoperative adhesions that may impair future fertility, increased size of surgical wounds, a longer hospital stay and recovery and possibly an increased risk of future placental previa/accreta $(8,12,19)$. Dilation and curettage should not be considered as the first choice of therapy. This is because the majority of the villi are implanted in the myometrium and it seems unlikely that the gestational sac can be expelled by curettage without perforating the uterine wall or damaging

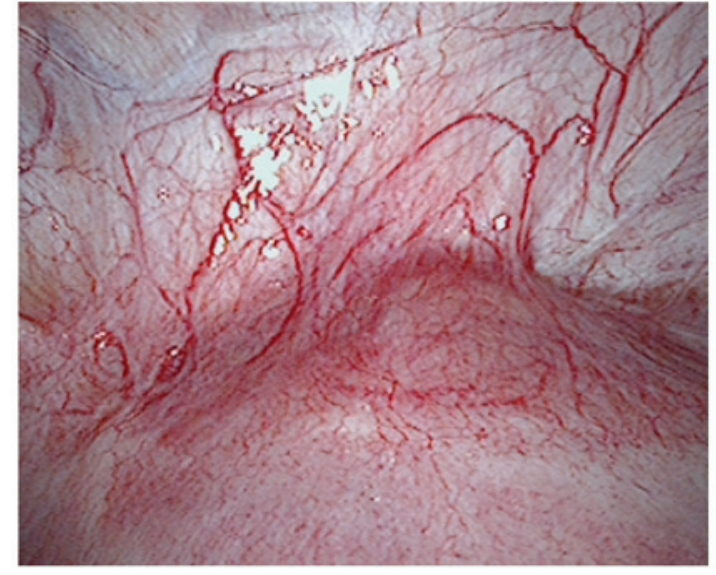

Figure 4. Laparoscopic image of CSP showing a topical purple bulge in the lower segment over the caesarean scar from a patient with CSP. CSP, caesarean scar pregnancy.

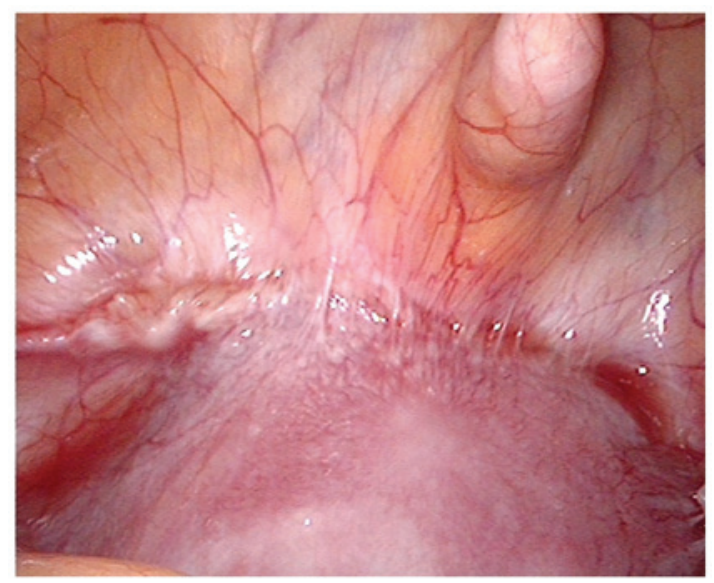

Figure 5. Laparoscopic image of CSP showing the uterine segment with some large adhesions together with the surrounding tissues and caesarean scars from a patient with CSP. CSP, caesarean scar pregnancy.

the urinary bladder and may also cause life-threatening haemorrhage $(8,12)$. Isolated suction curettage was used as the initial treatment on five of our patients. Four patients suffered life-threatening haemorrhage and one patient suffered uterine damage that finally led to hysterectomy. Previous findings 
suggest that selective UAE can temporarily block uterine perfusion and minimize hemorrhage and they have used this technique before or after curettage (21).

UAE treatment of CSP was first reported in 1999 and has been used widely to control haemorrhage and preserve the uterus $(22,23)$. However, isolated UAE without eliminating the gestational sac results in the gradual decrease of serum $\beta$-HCG levels and irregular menstrual bleeding $(7,12)$. Therefore, uterine curettage should be performed after UAE. Gelfoam appears to promote clotting via physical effects by supporting thrombus development. Vascular occlusion with gelfoam causes acute necrotizing arteritis. This inflammatory process eventually leads to breakdown of the gelfoam within 1-3 weeks after embolization, with subsequent vascular recanalization (24). Because of its temporary nature, uterine curettage should be carried out as soon as possible. In published studies, uterine curettage was usually carried out within 24-72 h after UAE (20). In accordance with our study, the results demonstrated that UAE followed by uterine curettage may be an effective and safe treatment for CSP. In the pathological sample of CSP, trophoblast cells were found to have invaded the myometrium, adhered, implanted and even penetrated the myometrium (13). For this reason, $50 \mathrm{mg}$ of MTX was given by infusing the arterial catheter to manage the possibility of residual villi in the scar tissue. Previous studies reported that MTX alone followed by suction curettage requires more hospitalization time and causes greater bleeding volumes compared with UAE (9). Therefore, curettage after UAE combined with MTX (50 mg) is a safe and effective means of treating CSP.

Of note, suction curettage may also be used to treat CSP, unlike ordinary curettage, because ultrasonography of CSP displays a thinned or absent myometrium between the gestational sac and bladder ( $<5 \mathrm{~mm}$ in two-thirds of cases). Thus, careful monitoring of curettage is necessary to avoid damaging the uterus. Compared with laparoscopy-guided and ultrasound-guided curettage, vaginal bleeding and length of stay in hospital showed no significant difference, although serum $\beta$-HCG levels decreased more rapidly with ultrasound-guided curettage than with laparoscopy-guided curettage. The rate of decline was $84.3 \pm 5.5$ and $76.3 \pm 10.2 \%$, respectively. The reason may be that the sonogram shows more clearly the location of the gestational sac and thickness of the myometrium between the gestational sac and bladder. When curettage is monitored by ultrasound, the operators can remove the ectopic sac under direct vision and have clear views of the endometrium and myometrium. The advantage of laparoscopy-guided curettage is observing the appearance of the uterus under direct vision during exploration. The topical purple bulge was usually seen in the lower segment over the caesarean scar. The blood vessels show hyperplasia in the same area (Fig. 4). However, in the current study, eight of the laparoscopy-guided cases showed the uterine segment had some heavy adhesions with the surrounding tissues and caesarean scars were not easy to expose (Fig. 5).

Separating the adhesion may increase the risk of tissue damage. Furthermore, laparoscopic surgery may require anaesthesia and increase the costs and risk of surgery. Therefore, we considered whether ultrasound-guided curettage may be appropriate in women who are hemodynamically stable with an unruptured CSP of a myometrial thickness of $>2 \mathrm{~mm}$. Medical treatment including local, systemic and combined treatment with MTX may be appropriate in women with an unruptured CSP of $<8$ weeks' gestation and a myometrial thickness of $<2 \mathrm{~mm}(6,12)$.

There is no consensus on time period required for the following pregnancy or the risk of future pregnancies. The present study results indicated that, none of the patients had normal intrauterine pregnancies during the follow-up period, although the patients were followed up for only one year and the long-term consequences of the treatment are unknown. A recent study of 24 women successfully treated for prior CSP without surgical correction of the scar reported favourable reproductive outcomes and a recurrence rate of only $5 \%$ (17). It showed that $88 \%$ of woman conceived naturally, and $95 \%$ of the pregnancies were intrauterine while $65 \%$ appeared normal. Thirty-five percent ended in spontaneous abortion. Findings of that study showed that, fertility was successfully preserved in the women who opted for first-trimester termination of CSP (17). However, the findings require further validation in a larger group of cases.

In conclusion, CSP is an uncommon but dangerous occurrence because of the increased risk of uterine rupture and massive haemorrhage. The precise localization of early pregnancies by sonography and early recognition of the typical sonographic findings are critical. In diagnosing CSP, TVS and TAS should be used in combination to obtain an accurate diagnosis. Based on the present findings it may be concluded that UAE combined with MTX followed by ultrasound-guided curettage may be an effective and safe treatment for CSP. More importantly, emergency UAE should be recommended as the first choice to treat uncontrollable massive haemorrhage of CSP and preserve the uterus and future fertility.

\section{Acknowledgements}

The present study was supported by the Joint Funds of National Key Clinical Departments Foundation of China. The authors thank Mrs. Ruilian Chen and Dr Daqing Ma for their help and critical comments during manuscript preparation.

\section{References}

1. Larsen JV and Solomon MH: Pregnancy in a uterine scar sacculus: an unusual case of postabortal hemorrhage. S Afr Med 53: 142-143, 1978.

2. Tinelli A, Tinelli R and Malvasi A: Laparoscopic management of cervical-isthmic pregnancy: a proposal method. Fertil Steril 92: 829.e3-829.e6, 2009.

3. Zhang B, Jiang ZB, Huang MS, Guan SH, Zhu KS, Qian JS, Zhou B, Li MA and Shan H: Uterine artery embolization combined with methotrexate in the treatment of cesarean scar pregnancy: results of a case series and review of the literature. J Vasc Interv Radiol 23: 1582-1588, 2012.

4. Köroğlu M, Kayhan A, Soylu FN, Erol B, Schmid-Tannwald C, Gürses C, Karademir I, Ernst R, Yousuf A and Oto A: MR imaging of ectopic pregnancy with an emphasis on unusual implantation sites. Jpn J Radiol 31: 75-80, 2013.

5. Seow KM, Huang LW, Lin YH, Lin MY, Tsai YL and Hwang JL: Cesarean scar pregnancy: issues in management. Ultrasound Obstet Gynecol 23: 247-253, 2004.

6. Maymon R, Halperin R, Mendlovic S, Schneider D, Vaknin Z, Herman A and Pansky M: Ectopic pregnancies in Caesarean section scars: the 8 year experience of one medical centre. Hum Reprod 19: 278-84, 2004. 
7. Sadeghi H, Rutherford T, Rackow BW, Campbell KH, Duzyj CM, Guess MK, Kodaman PH and Norwitz ER: Cesarean scar ectopic pregnancy: case series and review of the literature. Am J Perinatol 27: 111-120, 2010

8. Jurkovic D, Hillaby K, Woelfer B, Lawrence A, Salim R and Elson CJ: First-trimester diagnosis and management of pregnancies implanted into the lower uterine segment Cesarean section scar. Ultrasound Obstet Gynecol 21: 220-227, 2003.

9. Zhuang Y and Huang L: Uterine artery embolization compared with methotrexate for the management of pregnancy implanted within a cesarean scar. Am J Obstet Gynecol 201: 152.e1-152.e3, 2009.

10. Sugawara J, Senoo M, Chisaka H, Yaegashi N and Okamura K: Successful conservative treatment of a cesarean scar pregnancy with uterine artery embolization. Tohoku J Exp Med 206: 261-265, 2005.

11. Fylstra DL: Ectopic pregnancy within a cesarean scar: a review. Obstet Gynecol Surv 57: 537-543, 2002.

12. Ash A, Smith A and Maxwell D: Caesarean scar pregnancy. BJOG 114: 253-263, 2007.

13. Wang CB and Tseng CJ: Primary evacuation therapy for Cesarean scar pregnancy: three new cases and review. Ultrasound Obstet Gynecol 27: 222-226, 2006.

14. McKenna DA, Poder L, Goldman M and Goldstein RB: Role of sonography in the recognition, assessment, and treatment of cesarean scar ectopic pregnancies. J Ultrasound Med 27: 779-783, 2008

15. Maymon R, Halperin R, Mendlovic S, Schneider D and Herman A: Ectopic pregnancies in a Caesarean scar: review of the medical approach to an iatrogenic complication. Hum Reprod Update 10: 515-523, 2004.
16. Vial Y, Petignat P and Hohlfeld P: Pregnancy in a cesarean scar. Ultrasound Obstet Gynecol 16: 592-593, 2000.

17. Ben Nagi J, Ofili-Yebovi D, Marsh M, Jurkovic D and Jurkovic D First-trimester cesarean scar pregnancy evolving into placenta previa/accreta at term. J Ultrasound Med 24: 1569-1573, 2005.

18. Osborn DA, Williams TR and Craig BM: Cesarean scar pregnancy: sonographic and magnetic resonance imaging findings, complications, and treatment. J Ultrasound Med 31: 1449-1456, 2012

19. Wang YL, Su TH and Chen HS: Laparoscopic management of an ectopic pregnancy in a lower segment cesarean section scar: a review and case report. J Minim Invasive Gynecol 12: 73-79, 2005.

20. Wu X, Zhang X, Zhu J and Di W: Caesarean scar pregnancy: comparative efficacy and safety of treatment by uterine artery chemoembolization and systemic methotrexate injection. Eur J Obstet Gynecol Reprod Biol 161: 75-79, 2012.

21. Jiao LZ, Zhao J, Wan XR, Liu XY, Feng FZ, Ren T and Xiang Y: Diagnosis and treatment of cesarean scar pregnancy. Chin Med Sci J 23: 10-15, 2008.

22. Akbayir O, Gedikbasi A, Akyol A, Ucar A, Saygi-Ozyurt S and Gulkilik A: Cesarean scar pregnancy: a rare cause of uterine arteriovenous malformation. J Clin Ultrasound 39: 534-538, 2011.

23. Liang F and He J: Methotrexate-based bilateral uterine arterial chemoembolization for treatment of cesarean scar pregnancy. Acta Obstet Gynecol Scand 89: 1592-1594, 2010.

24. Jander HP and Russinovich NA: Transcatheter gelfoam embolization in abdominal, retroperitoneal, and pelvic hemorrhage. Radiology 136: 337-344, 1980. 